\title{
Especial
}

\section{Problemática multifactorial del absentismo laboral, el presentismo y la procrastinación en las estructuras en que se desenvuelve el trabajador}

\section{Multifactorial problem of presenteeism and absenteeism procrastination in structures in which the worker develops}

\author{
Marcos de Antonio García \\ Unidad de Ergonomía. Madrid. España \\ Recibido: 26-01-11 \\ Aceptado: 03-06-11

\section{Correspondencia} \\ Marcos de Antonio García \\ Urb. Prado Largo, 63 \\ 28223 Pozuelo de Alarcón (Madrid). España. \\ Tfno: 913520804 \\ E-mail:marcosdeantonio@yahoo.es
}

Universidad Complutense. Escuela Nacional de Medicina del Trabajo.

Resumen

Se analizan los conflictos psicosociales, como causa de deterioro de las relaciones laborales de los trabajadores, que producen tasas altas de absentismo, presentismo y/o procrastinación.

Med Segur Trab (Internet) 2011; 57 (223) 111-120

Palabras clave: conflictos psicosociales, absentismo, presentismo, procrastinación.

Abstract

It discusses the psychosocial conflicts as a cause of deterioration oflabor relations workers, who produce high rates of absenteeism, presenteeism and procrastination.

Med Segur Trab (Internet) 2011; 57 (223) 111-120

Keys word: psychosocial conflicts, absenteeism, presenteeism, procrastination. 


\section{INTRODUCCIÓN}

En España, dentro de los acuerdos que se suscriben entre Sindicatos, Patronal y Gobierno se define el absentismo laboral, como toda ausencia de una persona de su puesto de trabajo, en horas que correspondan a un día laborable, dentro de la jornada legal de trabajo ${ }^{1}$. El absentismo es uno de los costes que más preocupan a las empresas y que más tratan de controlar y reducir.

\section{Factores psicosociales del absentismo laboral}

El Absentismo Laboral produce una serie de conflictos psicosociales a consecuencia del deterioro significativo de las relaciones laborales de los trabajadores que producen tasa alta de absentismo creando una problemática multifactorial, en las estructuras en que se desenvuelve el trabajador: laboral, social y económica.

Laboral: El absentismo laboral crece ante la fatiga, las tareas monótonas, repetitivas y parcializadas que disminuyen, considerablemente, el interés y la motivación, y que le llevan a una situación de alienación, de la que intenta evadirse. De este tema se ocupa la Ciencia Ergonómica que debe de estar bien prestigiada y estructurada dentro de la Empresa.

Económica: El absentismo laboral aumenta cuando el trabajador se enfrenta con que la relación remuneración-trabajo no le es en absoluto satisfactoria, lo cual le conduce a una mayor desmotivación en la realización de sus tareas.

Social: El absentismo laboral disminuye cuando el trabajador se enfrenta ante el paro o la crisis económica, y se esfuerza en reducir la tasa de absentismo, como instinto primario de conservación; el problema es que esta situación está dando lugar a una nueva forma de absentismo que comentaremos más adelante. En referencia a los tipos o formas en que un empleado se ausenta de su puesto de trabajo, se los enumera de tres maneras:

a) El absentismo previsible y justificado. Es aquel que puede ser controlado porque la empresa está informada previamente de la ausencia, y se produce por causas reconocidas legalmente: Incapacidad temporal (I.T.), los relacionados con la actividad sindical, etc).

a1. El coste empresarial puede ser muy elevado

Como costes directos, citemos el del complemento salarial, el del sustituto, el de la seguridad social y el que implican los accidentes de trabajo.

Como costes indirectos, enumeremos el tiempo que implica el buscar a la persona adecuada para sustituir al empleado, la formación que hay que darle a esa persona, la adaptación del sustituto al puesto de empleo, la aceptación del sustituto por parte de sus compañeros y la recarga de tareas que implica para el resto de los empleados, entre otras cosas.

a2. Control de la situación de I.T. por parte del empresario

Junto con la posibilidad del empresario de instar la actuación de las inspecciones de los servicios médicos públicos, a través de emisión de informes, o denuncias respecto a la actuación del trabajador que está en situación de I.T., el art. 20.4 del Estatuto de los Trabajadores permite a las empresas verificar el estado de enfermedad o accidente del trabajador que se ha alegado por éste para justificar sus faltas de asistencia al trabajo, mediante reconocimiento a cargo de personal médico. Este precepto persigue el contrarrestar el absentismo laboral, y el posible fraude en la percepción del subsidio o el control de las faltas de asistencia al trabajo debidas a dicha situación.

- La empresa puede encargar la verificación del estado del trabajador a través del médico de empresa o facultativo elegido por ésta. 
- Las funciones de control de la empresa quedan limitadas a constatar el estado de salud del trabajador, sin que en ningún caso pueda acordar el alta, por lo que, ante la disparidad de criterios entre el médico designado por el empresario y los del servicios público, e incluso ante las sospechas de una conducta fraudulenta por parte del trabajador, el camino a seguir por la empresa será acudir a la inspección médica del servicios público correspondiente, para formular una denuncia o queja.

- Si el trabajador se negara a someterse a los reconocimientos médicos impuestos por la empresa, tendrá como consecuencia, la pérdida de los complementos o mejoras económicas de I.T. que estuviera gozando.

- Algunos sectores doctrínales consideran, que el empresario podrá suspender el abono del subsidio económico de los días $4 .^{\circ}$ al $15^{\circ}$ de la baja.

- La jurisprudencia ha admitido que se empleen medios distintos de la medida de reconocimiento médico para controlar comportamientos contrarios a la buena fe por parte del trabajador (STS 23-1-1990). Tal sería el caso, por ejemplo, de seguimiento a través de un detective.

Ante este escenario, algunos sectores abogan por conceder la competencia al médico de empresa, para controlar la baja laboral del trabajador, incluyendo la posibilidad de que su dictamen resultase vinculante para la inmediata alta del trabajador pseudo-enfermo. El gasto sanitario y económico que el absentismo supone para el erario público y el empresario, es de tal magnitud, que difícilmente se comprende la no adopción de medidas gubernamentales, que sin duda ahorrarían miles de millones de euros anuales

b) Absentismo no previsible y sin justificación. Se trata del abandono del puesto de trabajo sin autorización de la empresa (retrasos, salir a fumar, recados, cuidado de los hijos o de personas mayores, etc.).

En general estos absentismos pueden contribuir a reducir la productividad de una empresa, provocarle serios problemas organizativos y generarle elevados costes, ya que hay que cubrir el puesto del absentista, su ausencia puede provocar que haya maquinaria que cese su actividad, etc.

Lo que resulta evidente es que ante el absentismo laboral, las medidas prácticas a las que normalmente ha recurrido la empresa cuando se trata de reducir el excesivo absentismo son: aumento de severidad en los controles sanitarios, agravamiento de las sanciones en los casos de las ausencias no justificadas e instauración de premios antiabsentismo, y aunque es claro que estos métodos, en algunos casos tengan como efecto la disminución del número de jornadas perdidas, a medio y largo plazo, no son los medios adecuados para resolver verdaderamente el problema del que el absentismo es manifestación, ya que el resultado es que no se resuelve la frecuencia. Es evidente que su eficacia no es más que aparente, ya que la acción se dirige al síntoma y no contra la causa; es de esperar que la causa encuentre otra vía de manifestación, ya que resiste a la represión del síntoma y su consecuencia, por tanto pueden ser más dañinas (por ejemplo, aumento de los accidentes, aumento de los índices de desechos industriales, posible incremento de averías en maquinaria, disminución de la productividad, etc.). En este sentido, es interesante conocer las formulaciones realizadas por la experiencia laboral, recogida tanto por los investigadores sociales, como por los sindicatos, que se centran en cuatro grupos de factores nocivos:

1. Aquellos que pueden medirse con instrumentos objetivos, y por lo tanto, observar las condiciones de aceptabilidad o no en que se desenvuelve el trabajo, por ejemplo: la intensidad de la luz (con fotómetro), el ruido (fonómetro), la temperatura (termómetro), la humedad (higrómetro), la ventilación (anemómetro), etc. 
2. ${ }^{\circ}$ Se refiere a sustancias químicas existentes en el medio de trabajo introducidas, cada vez más, por las exigencias del proceso productivo (ejemplo: las fibras sintéticas utilizadas en la industria textil, las sustancias utilizadas en la producción de la goma sintética, las combinaciones de silicio orgánico, los productos del petróleo, los utilizados en la industria farmacéutica, el gas de sulfuro de carbono, los humos ácidos, etc.) en general: los polvos, gases, vapores o humos no solo tóxicos, sino también molestas, haciendo necesaria una evolución periódica con objeto de proteger la salud, y la higiene el bienestar del trabajador

3. ${ }^{\circ}$ Fatiga física y mental, porque la concentración mental y la tensión emotiva acompañan siempre al esfuerzo físico prolongado y porque la fatiga nerviosa y mental tienen siempre consecuencias sobre el rendimiento muscular. La fatiga de actividad física o muscular aparece más rápidamente cuando la contracción del músculo es de tipo estático. La actividad muscular requiere un cierto tiempo de preparación que está en relación con la dificultad del trabajo y con el entrenamiento.

El trabajo físico es causa de fatiga, y hasta un cierto límite, se puede considerar normal, y, por tanto, fisiológica. Más allá de este límite resulta excesiva pudiendo llegar a ser patológica. Pero la definición del límite entre fatiga fisiológica y fatiga patológica es tanto más difícil por cuanto se encuentra condicionada por innumerables factores. La fatiga patológica puede ser considerada como la consecuencia última de una situación ambiental que supera la capacidad de adaptación del individuo".

4..$^{\circ}$ El cuarto grupo de factores nocivos comprende aquellas condiciones de trabajo, distintas al trabajo tísico, capaces de provocar efectos de cansancio, por ejemplo: monotonía, ritmos excesivos, saturación de los tiempos, repetición, ansiedad, responsabilidad, actitudes de desacuerdo, etc.; Esta problemática está más presente allí donde se aplica la parcelación y simplificación de las operaciones, con reminiscencias taylorista sobre tiempos, ritmos de ejecución y pausa predeterminadas en contraposición con la cadencia espontánea del comportamiento individual y la cadencia de tiempos individuales.

Es posible adjetivar este conjunto de factores mostrados como capaces de provocar "efectos de cansancio", distintos a la fatiga física. Los "efectos de cansancio" no deben superar el límite que representa para el hombre el vivir una vida social completa dentro y fuera de la fábrica, en el marco temporal del día de la semana, del año y de la vida entera".

\section{El presentismo laboral}

A los dos tipos de absentismo, (absentismo previsible y justificado, y el absentismo no previsible y sin justificación) que hemos contemplado en nuestro análisis de la publicación anterior hay que sumarle un tercero muy de actualidad y especialmente perjudicial para la empresa.

El absentismo presencial o presentismo, consiste en acudir al trabajo, pero dedicando una parte de la jornada a actividades que no guardan relación con las tareas propias del puesto que se ocupa, entre las que se encuentran como más comunes consultar páginas web y usar el correo electrónico; Generalmente asociamos el absentismo presencial al uso de las nuevas tecnologías, pero en realidad su práctica es muy antigua, en otras épocas consistían en leer el periódico en horas de trabajo o hacer llamadas telefónicas o escribir a amigos y familiares, prácticas que aún hoy persisten.

\section{En el presente, el problema no es el absentismo laboral, el verdadero problema se encuentra en el presentismo}

En el 2009, el absentismo en España, que ostentaba unos porcentajes del 2`7, se ha conseguido reducir por debajo de la media europea, situándose en el 2,1\%, cuando la media europea se sitúa en $2,2 \%$. 
Pero esta reducción del absentismo no ha ido aparejada de una mejora de la productividad y del rendimiento laboral por que lo ocurrido en realidad es que hemos cambiado el absentismo laboral por el presentismo o "síndrome de la silla caliente"; que consiste en que se esta en el trabajo como si se trabajara pero sin hacerlo realmente; y es que. el miedo a perder el trabajo en la situación actual de crisis económica ha hecho que se reduzca de un modo muy significativo el índice de absentismo laboral. Las bajas laborales continuadas, la ausencia en el trabajo por unas horas, etc, se están reduciendo cada vez más, ya que los trabajadores saben de la difícil situación del mercado laboral y ya no se arriesgan a ser despedidos por estos motivos. Sin embargo, del presentismo laboral, surge una amenaza aún más inquietante para la empresa, por su difícil detección y por la merma de la productividad que en el absentismo justificado, e incluso en el injustificado.

Durante el presentismo laboral el empleado se encuentra en su puesto de trabajo, pero "ausente" en sus funciones, es lo que se conoce a nivel de los trabajadores que lo practican con el casticismo "tocarse los huevos en el curro". En realidad, en muchas ocasiones, este tipo de trabajadores están más horas presenciales que sus compañeros, con lo cual aparentan que tiene más trabajo que el que pueden abarcar en su horario normal. En época de crisis económica, es algo que se está convirtiendo en situación de alarmante frecuencia empresarial, ya que los empleados que pierden varias horas de su jornada laboral en distracciones y problemas personales, además están más propensos a cometer errores que afectan la productividad, durante el tiempo que utilizan para dar salida a lo más urgente de sus tareas.

Según una encuesta entre más de 1000 trabajadores, realizada por la empresa de recursos humanos Randstad, son ellos mismos los que han identificado esta tendencia con su afirmación en el 45,8 \% de los encuestados.

Existen varios tipos de presentismo:

Uno es el ya comentado de alargar la jornada laboral innecesariamente por el mero hecho de aparentar.

Otro tipo es el que practica aquel trabajador que para ensalzar sus funciones, tarda más tiempo en resolver los problemas, cuando, a lo mejor, puede hacerlo en mucho menor tiempo, de este modo, realza las funciones que desempeña porque les añade un componente de dificultad y esfuerzo que en realidad no existe.

Por último, estaría el presentismo del empleado que se queja constantemente del volumen de trabajo que tiene, cuando en realidad, su productividad es muy baja.

Añadamos, sin ánimo de justificar estas prácticas, que en este momento de crisis, nuestro país se encuentra a la cabeza de la cantidad de horas trabajadas al día por convenio y legislación laboral, sin embargo a la cola de la productividad, lo cual incide en una cierta predisposición cultural a quedarse más horas en la oficina, los trabajadores no están buscando mejorar el rendimiento de la empresa, sino asegurar su puesto de trabajo, hecho que reconocen los propios trabajadores: el $60 \%$ admite que su productividad no se ha incrementado con la crisis, sin embargo, y a pesar del reconocimiento, la aparente paradoja consiste en que, desde que comenzó la crisis, la productividad española ha aumentado a un ritmo del $2,63 \%$ anual, sin que este hecho se haya producido en la Eurozona. Lo cual se explica por que España ha elevado su productividad a expensas de los ajustes de plantillas, y las políticas de reducción de costes.

¿Cómo se detecta el presentismo? Ante la sospecha de esta problemática es necesario evaluar los resultados individuales del trabajador, no en equipo, porque es muy posible que los compañeros de los "presentistas" se estén cargando con el trabajo de estos. 


\section{Pautas para evitar el presentismo}

La falta de motivación está detrás de muchos casos de ausencia y presentismo de los trabajadores en las empresas. Una adecuada política de motivación es, sin duda, la mejor arma para luchar contra el absentismo. Para aplicarla es necesario averiguar qué medidas pueden contribuir a aumentar la satisfacción de los empleados e implantar acciones concretas. Una de las más valoradas es la flexibilidad horaria, ya que ayuda a compaginar la vida familiar y laboral. Algunos organismos y asociaciones alerta de los males de los horarios interminables que padecemos los españoles cuyos consecuencias son: a) baja productividad y alta siniestralidad laboral, a causa de que no se duerme lo suficiente; b) baja natalidad al no tener ni tiempo de vivir en pareja; c) fracaso escolar por falta de atención a los hijos, y 4) de esta situación de horarios irracionales, la mujer es la más perjudicada. También se identifican como posibles causantes la falta de promoción y de motivación de los empleados, y el hecho de que quizás realizan tareas monótonas en sus puestos de trabajo, y se destaca el que los empleados estén conectados a los objetivos generales y al proyecto de la empresa, siendo de gran transcendencia el hecho de que exista un vínculo emocional entre el trabajador y la empresa. También puede ser efectiva una adecuada de política de asistencia sanitaria que agilice la atención médica a los empleados y gracias a ello se reduzca el numero de días de absentismo laboral.

Otra cuestión a tener en cuenta es la utilización de técnicas de control informática por parte de la empresa, con el fin de coartar la libre navegación del trabajador, Está comprobado que recortar el acceso a Internet puede aumentar la productividad un $30 \%$, así que no es de extrañar que existan cada vez más filtros a miles de páginas de Internet dentro de las empresas. Hasta ahora, las grandes corporaciones han sido muy tolerantes con los empleados que han sido descubiertos usando Internet en asuntos no relacionados con su trabajo o en sitios web que podrían poner en riesgo a la empresa, pero algo había que hacer, y la cuestión es, ¿qué se puede hacer?

La gran disyuntiva se presenta a la hora de regular y de poner control en el uso indebido de la red.

De hecho las compañías invierten cada vez más en sistemas de control de redes y de usuarios y los instaladores están desarrollando sistemas en función de la seguridad con el fin de coartar la libre navegación del trabajador. Hay herramientas divididas por categorías, que, por ejemplo: prohíben la entrada a pornografía, búsqueda de empleo, revistas, etc. Cada empresa puede elegir que bloques desea vetar. Se ha llegado al extremo de utilizar el software diseñado por Microsoft donde se propone espiar al trabajador. También hay empresarios que contratan a expertos para que "espíen" la actividad informática de sus empleados y les informe sobre el quehacer de sus trabajadores.

No obstante el empresario debe de preguntarse ¿Cambia el empleado su comportamiento con la aplicación de estos sistemas?. Aunque no he encontrado estudios sobre el tema en nuestra realidad nacional, la implementación de medidas de seguridad o de filtros es fácil entender que pueden llegar a producir el efecto contrario, ya que un empleado que se siente controlado puede también sentirse acosado, lo que podría derivar en desmotivación, falta de compromiso y en una merecida desconfianza hacia su empresa, que es justo lo contrario de lo que en principio se debería de buscar. En nuestra filosofía laboral está profusamente extendida la idea que se comprime en aquella frase de "normas de cumplimiento = normas de no cumplo y miento".

Por ahora, parece que lo único que las empresas pueden y debe de hacer es explicar al empleado la normativa sobre el acceso a Internet, que la misma se ponga por escrito y que ambas partes la firmen. En este caso, las empresas avisan al empleado tras lo cual pasan a su control (lo cual es lo legal). Así que la empresa deberá especificar que ese control se está aplicando para evitar el presentismo.

Pero este control también tiene unos límites, el empleado tiene sus derechos ya que la incorporación de estos métodos podría chocar con el derecho a la intimidad de los 
empleados..., y entonces ¿cómo queda la empresa?. Según la información disponible, el Tribunal Supremo dictó que: "sólo se puede registrar el ordenador de un empleado si la empresa establece una normativa expresa”. Así que en caso de juicios los límites sobre el control al empleado los definen las sentencias de los tribunales como cualquier otro litigio y quedan sometidos a las interpretaciones de las leyes.

\section{La procrastinación}

¿Que es la procrastinación. ¿No lo sabe?, ¿ Seguro?, Piense un poco..., recuerda sus tiempos de estudiante, en los que con cualquier excusa retrasabas el estudio o tarea obligatoria, excusas la mayoría de las veces tan peregrinas como que estaba a punto de pasar de nivel en ese video juego que quizás no fuera su preferido... pero cualquier cosa nos servía de excusa para alargar el momento de hincar los codos, ante aquel peñazo del que se iba a examinar uno en unos días (o incluso el día siguiente).

\section{La procrastinación: una epidemia de nuestros días}

La procrastinación es un complejo transtorno del comportamiento que a todo el mundo nos afecta en mayor o menor medida. Consiste en postergar de forma sistemática aquellas tareas que debemos hacer, que son cruciales para nuestro desarrollo y que son reemplazadas por otras más irrelevantes pero más placenteras de llevar a cabo. Es asumida popularmente como simple "pereza", aunque en realidad no es exactamente pereza: es el realizar trabajos menos pesados para evitar los que deberíamos estar haciendo, y así justificar que estamos muy ocupados para ocuparnos de el en ese momento.

Eso es procrastinación, una palabra que condensa el famosísimo refrán "no hagas hoy lo que puedas dejar para mañana" y que pese a no ser ampliamente utilizada en nuestro vocabulario, no cabe la menor duda que todos, en alguna medida, hemos "procrastinado" un poco, ¿o bastante?. Afecta a multitud de perfiles, además del de el ejemplo expuesto sobre el estudiante que aplaza indefinidamente el estudiar para sus exámenes,etc.), también (el ejecutivo que aplaza una y otra vez una reunión que prevee conflictiva.

\section{¿Que produce la Procrastinación?}

Se han propuesto seis fuentes de procrastinación en función del tipo de dudas que nos frenan para el inicio o la progresión de la tarea.

1. Dudas en realizar tareas arduas que no disfrutamos.

Las tareas extensas o complejas ocasionan desgana. No se encuentra placer en ello, y existen un millón de tareas alternativas que se preferiría abordar. Ejemplo: El esposo que, hubiese aplazado el corte del césped hasta que se convierta en una jungla. Sabía que cortar el césped asearía mi casa y complacería a la esposa, pero aborrecía prestarle tiempo y atención a esa tarea.

2. Dudas en encarar actividades para las que carecemos de habilidad.

Es posible que se disfrute de las artes, e incluso nos fascinen las personas altamente creativas. Sin embargo, si no estamos particularmente dotados para la pintura el dibujo o el diseño. Es por ello que podría evitarse comenzar la realización de la decoración. Por ejemplo: Disfruto de un refinado trabajo de poda y decoración de un árbol de navidad o una habitación elegantemente decorada, pero considero que carezco de talento para embellecer las cosas. Como consecuencia de ello aplazo, lo relacionado con su decoración lo más posible.

3. Dudas en hacer lo que no está programado en nuestra agenda.

La naturaleza humana tiende a moldearse a si misma y adquirir una percepción homogénea, subjetiva y hierática. Cuando las circunstancias cambiantes tornan en obsoletos nuestros planes, nos resistimos a severos ajustes. Tozudamente, nos 
aferramos a nuestra visión del curso de acción programada y evitamos alterar nuestra conducta.

4. Dudas en solucionar situaciones confusas

Un ejemplo de base es la confrontación. Es difícil decirle a alguien algo que no quiere escuchar y, muy probablemente, el interlocutor no reaccionará bien a nuestra sugerencia. De forma que por miedo al conflicto, procrastinamos o dilatamos la situación en vez de iniciar la confrontación.

5. Dudas en enfrentar una tarea que requiere de elevada energía y nos proporciona poco reconocimiento.

En calidad de líderes podríamos estar tentados en tener la mayor rentabilidad de cada uno de nuestros euros invertidos. Lo mismo ocurre con nuestro tiempo. Lo queremos invertir de la manera más rentable. De forma que podrían surgirnos dudas respecto de asignar parte de nuestra energía en relaciones que requieren un elevado mantenimiento o requieren de un contacto recurrente con el inversor o el cliente, que no es contribuidor sustancial de la organización.

6. Dudas en enfrentar asuntos que no comprendemos

Existe un dicho que reza, "No se puede enseñar trucos nuevos a un perro viejo." Y nuestra generación ha debido incorporarse a tecnologías de continua innovación, $y$ no ha sido fácil. Probablemente no entendemos muchas características de los sistemas GPS, iPods, o PDA, y, como resultado, aplazamos el hecho de incorporarlos a nuestras vidas profesional o cotidiana -inclusive a sabiendas que serían muy útiles para nosotros-.

La cuestión es que y cada vez más esta asunto de la procrastinación se está convirtiendo en un serio de problema que afecta a al salud psicológica de los individuos, al efectivo funcionamiento de las empresas y, por ende, a la salud social de una comunidad.

La procrastinación es un fenómeno que se ha descubierto de tal complejidad que resulta difícil analizarlo, por las complicaciones que presenta en identificar sus orígenes así como las muchas relaciones causa-efecto que se realimentan entre sí. Todo esto dibuja un cuadro polifacético que resulta muy complejo de analizar. Sin embargo en el ámbito de la literatura científica sobre el tema existe un común denominador que concluye, que la procrastinación se manifiesta ante todo como "una pésima gestión del tiempo".

Cuando "procrastinamos" Solemos o bien sobrestimar el tiempo que nos queda para realizar una tarea, o bien subestimar el tiempo necesario —según los recursos de que disponemos - para realizarla. Éstos son solamente un par de los muchos autoengaños en los que el incurrimos los procrastinadores.

De hecho, una de las actitudes típicas de un perfil determinado de procrastinador es la excesiva autoconfianza., una falsa sensación de autocontrol y seguridad. Por ejemplo, imaginen que se nos da 15 días para presentar un informe. En nuestro fuero interno estamos convencidos que solo necesitaremos 5 días para hacerlo, incluso menos. En ese momento pensamos "¡hay tiempo de sobra, no es necesario ni siquiera empezar a bacerlo tan pronto!". Y se posterga día tras otro una tarea que no solamente no nos ilusiona hacer, si no que, en cierta manera "ya hemos terminado" en nuestra mente confiada, cuando ni siquiera hemos movido un dedo por ella. Al acercarse el plazo de entrega de forma peligrosa, de repente, nos damos cuenta de que no seremos capaces de cumplir con la tarea que se nos ha asignado. Entonces pensamos “¡No tengo esto bajo control, no tendré tiempo!" y comenzamos a trabajar en ello de forma atropellada, con una gran carga de estrés. En ese momento aparece en escena otro autoengaño, y es el de qué: “Bueno, no importa, en realidad solo bajo presión trabajo bien!”; Lógicamente, porque ya no hay otra opción en ese punto. Frecuentemente esta actitud y manera de proceder es típica de personas que confían mucho en sus posibilidades. Si, además, es realmente así - la persona tiene realmente esas capacidades- es posible que el final de la historia sea que aquella tarea se entregue en el plazo y con unos resultados óptimos y esto envía un mensaje erróneo al procrastinador (“¡a fin de cuentas, qué buenos resultados 
has obtenido!") lo cual le nos incita a autoconfirmarnos que hemos obtenido una recompensa a la forma estresada de trabajar; por lo que insistiremos en nuestra conducta, aunque ésta siempre nos traiga ansiedad y problemas en general.

\section{¿Cuales son las causas que pueden llevar a una persona a padecer de procrastinación?}

Son diversas y complejas pero esquemáticamente se asocian con a estado depresivo (la depresión conduce a estados de letargo), también al perfeccionismo, (evitan empezar proyectos nuevos porque temen que no poder alcanzar la perfección que desean, lo que hace que pierden la motivación). También la baja tolerancia a la frustración (esta, ayuda a "dejar las cosas de lado", por miedo a verse desbordado y el consecutivo temor miedo a sentirse anímicamente mal). Otro perfil muy distinto sería el de aquellas personas muy activas que disfrutan gestando ideas, pero que no pueden finalizarlas porque enseguida se distraen generando la siguiente; y postergan así decenas de tareas que no tienen tiempo para completar, Esto obviamente conduce a una forma de procrastinación, muy común entre las personas especialmente creativas que en no pocas ocasiones se reconduce trabajando coordinadamente con otras personas en las que domine la inteligencia ejecutiva. Y eso solo mencionando una minúscula porción de los muchos perfiles de procrastinador que se pueden encontrar. Seguramente usted, que está leyendo estas líneas se haya visto identificado en alguna de las frases de este artículo. Se habrá recordado a sí mismo leyendo el diario en la oficina con una lista de tareas por hacer, yendo a la cafetería justo en el instante en que se propuso empezar un proyecto, navegando por Internet mientras su teléfono sonaba con un cliente incómodo al otro lado, etc. El hecho de que sea un mal muy extendido y que se trate de un fenómeno de por sí fascinante por su complejidad y riqueza de matices, hace que merezca la pena su estudio, tanto a nivel académico, que ya se viene realizando, como a nivel individual, empresarial y colectivo.

\section{¿Como afrontar la procrastinación?}

La solución no es fácil, y las empresas poco o nada pueden hacer en estos casos, salvo divulgar el conocimiento sobre la procrastinación así como las técnicas más aceptadas y útiles para que sepa y pueda combatirlas el propio trabajador, tales como: "Falsear" de forma consciente nuestras propias previsiones. Por ejemplo, si creemos que tardaríamos una semana en hacer algo, pues sistemáticamente duplicar o incluso triplicar ese tiempo en nuestra agenda. Así al menos, podríamos evitar defraudar a nuestros clientes, jefes, etc. Otra técnica que puede ser útil es desglosar una tarea en casi todas sus partes componentes, para hacer previsiones de tiempo lo más honestas posibles.

Por último describiremos la técnica para combatirla que descubrió la psicóloga Bluma Zeigarnik, mientras estaba tomando un té en una cafetería de Viena. Allí observó que los camareros recordaban fácilmente los pedidos de los clientes cuando éstos pedían la cuenta. Pero, tras pagar la cuenta, si al cliente preguntaba algo al respecto unos minutos después, entonces al camarero le costaba recordar lo consumido. Es decir, al pagar, el camarero parecía borrar el pedido de su mente.

Zeigarnik, inspirada, regresó al laboratorio para probar su idea.

Pidió a varias personas que realizaran algunas tareas sencillas (como apilar fichas o meter juguetes en una caja), pero, en algunos casos, detuvo a los participantes antes de que acabaran ciertas tareas. Al final del experimento, pidió a los participantes que describieran las tareas realizadas. Como en su observación de los camareros, Zeigarnik descubrió que las tareas sin finalizar quedaban grabadas en la mente de las personas y, por tanto, eran más fáciles de recordar.

Al iniciar cualquier tarea, nuestra mente experimenta una especie de ansiedad psíquica. Al concluirla, nuestra mente se relaja. Pero si no la concluimos, nuestra mente inquieta continúa importunando hasta que se termina lo iniciado. 
¿Cómo podemos aplicar esto para combatir la procrastinación? Como lo que de verdad nos abruma es realizar la tarea pesada, lo que podemos hacer es persuadirnos de que sólo llevaremos a cabo esa tarea durante unos minutos. Sólo unos minutos no hacen daño a nadie. A menudo, entonces, sentiremos la necesidad de seguir con ella hasta acabarla.

Las investigaciones demuestran que la regla de "sólo unos minutos" es muy eficaz para vencer la procrastinación y puede ayudar a terminar las tareas más arduas.

\section{REFERENCIAS BIBLIOGRÁFICAS}

1. Regulación jurídica del absentismo laboral en el Estatuto de los Trabajadores de España aprobado por Real decreto Legislativo 1/1995, de 24 de marzo (B.O.E. 29/03/1995).

\section{BIBLIOGRAFÍA RELACIONADA}

Bauman, Zygmunt (1999). Modernidad líquida. Fondo de Cultura Económica. Buenos Aires.

Bauman, Zygmunt (2005). Vidas desperdiciadas: La modernidad y sus parias. Paidós Ibérica. Barcelona.

Beer, M. et al. (1989): Gestión de los Recursos Humanos, Ed. Ministerio del Trabajo, España.

Hemp, Paul. 2004. Presenteeism: At Work but Outof it, Harvard Business Review, Oct 2004.

Isaac Camacho, (2006): Una Defensa desde la Enseñanza de la Ciencia, Revista Eureka sobre la Enseñanza y divulgación de la Ciencia. Año/volumen. 3. numero 002. Asociación de Profesores Amigos de la Ciencia EUREKA. Cádiz, España. Pp. 220-228.

Marx C., (1975). Crítica del Programa de Gotha. Ed. Ciencias Sociales

McCrea, S.M., N. Liberman, Y. Trope \& S.J. Sherman. 2008. Construal level and procrastination. Psychological Science 19: 1308-1314.

Robbins, S. (2006): Comportamiento Organizacional teoría y práctica, 7 Edición ,Ed. Prentice Hall Hispanoamericana,

Rojas, R., 2007. Enfoque del Presentismo en Empresas de Salud. Cienc y Trab. Abr-Jun; 9(24) 64-68.

Steel, P., 2007. The nature of procrastination: A meta-analytic and theoretical review of quintessential self-regulatory failure. Psychological Bulletin 133: 65-94.

Aledo M., Factores que inciden en el Clima Laboral. http://www.gerenteweb.com

Enebral J., En busca de la satisfacción y aun disfrute, en el desempeño profesional ;http://www.arearh. $\mathrm{com} / \mathrm{rrhh} / \mathrm{satisfaccion} . \mathrm{htm}$

González L., Los Estímulos Morales y Materiales en el Desarrollo Económico y la Formación del Hombre Nuevo.http://www.nodo50.org/cubasigloXXI/economia/grodriguez1_310904.htm

Maisch ; E., Pautas Metodológicas para la Realización de Estudios de Cima Organizacional ; http://www. getiopolis.com/canales $/ \mathrm{hr}$./1/opciones.htm

Tuban, J., Medición del Clima Laboral en las Organizaciones. http://www.tablero-decomando.com y www.monografias.com/trabajos6/medicli/medicli.shtml

(*) Doctor "Cum Laude" en Ciencias Sociales. Especialidad Universitaria Oficial en Psicología Social. Licenciado con M. de H. en Fisioterapia. Ex-Responsable del la Unidad de Ergonomía del INMST. Ex-Profesor Asociado de Biomecánica Ergonómica y Psicosociología Laboral en el Escuela de Medicina del Trabajo de la Facultad de Medicina de la U. C. M./Ex-Profesor Titular de Biomecánica y Cinesiología en la Escuela de Fisioterapia de MAPFRE, Universidad Pontificia de Salamanca en Madrid. Diplomado Oficial con M. de H. en Ergonomía. Jubilado. 